\title{
Plate Tectonics and Reality
}

\author{
Dharmaj Mitra
}

\section{Introduction}

Plate tectonics is the scientific theory that attempts to explain the movements of the Earth's lithosphere that have formed the landscape features we see across the globe today. By definition the word "plate" in geologic terms means a large slab of solid rock. "Tectonics" is a part of the Greek root for "to build" and together the terms define how the Earth's surface is built up of moving plates. The theory of plate tectonics itself says that the Earth's lithosphere is made up individual plates that are broken down into over a dozen large and small pieces of solid rock.

These fragmented plates ride next to each other on top of the Earth's more fluid lower mantle to create different types of plate boundaries that have shaped the Earth's landscape over millions of years.

\section{History Of Plate Tectonics}

Plate tectonics grew out of a theory that was first developed in the early 20th century by the meteorologist Alfred Wegener. In 1912, Wegener noticed that the coastlines of the east coast of South America and the west coast of Africa seemed to fit together like a jigsaw puzzle .Further examination of the globe revealed that all of the Earth's continents fit together somehow and Wegener proposed an idea that all of the continents had at one time been connected in a single supercontinent called Pangaea. He believed that the continents gradually began to drift apart around 300 million years ago - this was his theory that became known as continental drift.

The main problem with Wegener's initial theory was that he was unsure of how the continents moved apart from one another.

Throughout his research to find a mechanism for continental drift, Wegener came across fossil evidence that gave support to his initial theory of Pangaea. In addition he came up with ideas as to how continental drift worked in the building of the world's mountain ranges. Wegener claimed that the leading edges of the Earth's continents collided with each other as they moved causing the land to bunch up and form mountain ranges.

He used India moving into the Asian continent to form the Himalayas as an example.

Eventually Wegener came up with an idea that cited the Earth's rotation and its centrifugal force toward the equator as the mechanism for continental drift. He said that Pangaea started at the South Pole and the Earth's rotation eventually caused it to break up, sending the continents toward the equator. This idea was rejected by the scientific community and his theory of continental drift was dismissed as well.

In 1929, Arthur Holmes, a British geologist, introduced a theory of thermal convection to explain the movement of the Earth's continents. He said that as a substance is heated its density decreases and it rises until it cools sufficiently to sink again. According to Holmes it was this heating and cooling cycle of the Earth's mantle that caused the continents to move. This idea gained very little attention at the time.

By the 1960s, Holmes' idea began to gain more credibility as scientists increased their understanding of the ocean floor via mapping, discovered its mid-ocean ridges and learned more about its age. In 1961 and 1962, scientists proposed the process of sea floor spreading caused by mantle convection to explain the movement of the Earth's continents and plate tectonics.

\section{Principles Of Plate Tectonics Today}

Scientists today have a better understanding of the make-up of the tectonic plates, the driving forces of their movement, and the ways in which they interact with one another. A tectonic plate itself is defined as a rigid segment of the Earth's lithosphere that moves separately from those surrounding it.

There are three main driving forces for the movement of the Earth's tectonic plates. They are mantle convection, gravity, and the Earth's rotation. Mantle convection is the most widely studied method of tectonic plate movement and it is very similar to the theory developed by Holmes in 1929. There are large convection currents of molten material in the Earth's upper mantle. As these currents transmit energy to the Earth's asthenosphere (the fluid portion of the Earth's lower mantle below the lithosphere) new lithospheric material is pushed up toward the Earth's crust.

Evidence of this is shown at mid-ocean ridges where younger land is pushed up through the ridge, causing the older land to move out and away from the ridge, thus moving the tectonic plates. 
Gravity is a secondary driving force for the movement of the Earth's tectonic plates. At mid-ocean ridges the elevation is higher than the surrounding ocean floor. As the convection currents within the Earth cause new lithospheric material to rise and spread away from the ridge, gravity causes the older material to sink toward the ocean floor and aid in the movement of the plates. The Earth's rotation is the final mechanism for the movement of the Earth's plates but it is minor in comparison to mantle convection and gravity.

As the Earth's tectonic plates move they interact in a number of different ways and they form different types of plate boundaries. Divergent boundaries are where the plates move away from each other and new crust is created. Mid-ocean ridges are an example of divergent boundaries. Convergent boundaries are where the plates collide with one another causing the suduction of one plate beneath the other. Transform boundaries are the final type of plate boundary and at these locations the no new crust is created and none is destroyed. Instead the plates slide horizontally past one another. No matter the type of boundary though, the movement of the Earth's tectonic plates is essential in the formation of the various landscape features we see across the globe today.

Other reasons are:

1. Tidal drag due to the gravitational force of the moon ( and the sun) exerts on the crust of the earth.

2. Global deformation of the geoid due to small displacements of the rotational pole with respect of the earths crust.

3. Other smaller deformation effects of the crust due to wobbles and spin movement of the earth rotation on a small time scale.

Is Modern scientist's above mentioned determinations are correct?

Answer: No

What is the real cause for plate tectonics?

Answer: The melting ice of the earth.

Why?

There are another eight planets in our solar system. But there have no evidence for plate tectonics. The difference between Earth and other plants are that, in Earth have ice coverage and other planets have no ice coverage. Once, our Earth is covered by ice. While melting of this ice coverage from equator to pole, plate tectonics began. We must think about why before ice age, here have no plate tectonic phenomena?

\section{Summary}

The thick layer of Earth's crust is called Lithosphere. Once upon a time, Lithosphere was a single layer. Then it split into two, and later on split into seven parts. These parts are called plates. The plates are African plate, North American plate, South American Plate, Eurasian plate, Indo Australian plate and pacific plate. There are three more smaller plates known as Arabian, Nazca and Philippines. All these plates move in different directions with different speeds. The speed varies from $2 \mathrm{~cm}$ to $10 \mathrm{~cm}$ per year. In other words, their speeds of movement can be compared to the speed of growth of nails in our body. Around 300million years ago, the surface of earth was divided into two continents. One was Gondwana Land and the other, Laurasia. The present day plates the South America, Africa, India, Australia and Antarctica- were included in the Gondwane Land. Present day North America and Eurasia were combined together as Laurasia. Around 275 million years ago, Gondwane Land and Laurasia collided with each other to form a super continent called Pangea. During the last 170 millions of years, Pangea got divided into floating parts of different sizes. Due to the collision, Indo Australian plate penetrated in to Euratian plate at about $2000 \mathrm{~km}$. By this way instead of ice coverage, Himalayam and Tibatean platueau took place.

According to science, the reason behind the floating of these continental plates is the thrust by the molten lava present inside the earth. But, actually the plates do not move because of this reason. There are eight more planets like our earth, in the solar system. Each has molten lava and volcanic eruptions. But none of them have moving plates on their crusts. So, we can assume that there is some other reason, than the thrust by lava.

The real reason relates to the ice-mountains which covered earth, a long time ago. As we know, ice is solid, and a solid substance is under the influence of Earth's gravitational pull. This gravitational force is that which gives spherical shape to stars, planets and satellites. This force folds the flat-elongated parts of these bodies, so that they attain spherical shape. This results in the formation of plates and their movement.

Around 300 million years ago, surface of earth was covered by ice-mountains. As they started melting and moving away from the equatorial region, earth started losing its spherical shape. To regain the spherical shape from this egg-like shape, earth exerted extra-gravitational force along its North-South direction. This extra gravitational force caused the collision of Gondwana Land and Laurasia, and the drifting of plates in the Eastwest direction. 
The melting of ice from the equatorial region towards the poles, which started around 300 million years ago, still continues. Remaining stock of ice on earth is available only at the poles-at Arctic and at Antarctic. The drifting of plates will continue, till all the ice on the earth melts away.

As far as earth is concerned, it tends to perpetuate its spherical shape. The factor that changes its spherical shape is the presence of ice. Solar heat is that, which changes the form of ice. When the luminosity of sunlight was 0.7 , the whole of earth's surface was covered with ice. Gradually the luminosity increased to 1 and as a result, ice at the equatorial region started melting. As the luminosity reaches 1.9 , there will be no ice left on earth. By then, the drifting of plates and the resulting earthquakes will cease. Now plates drift in the East-West direction. From this fact, we can assume that the reason for this drift is the gravitational pressure exerted on the equatorial region by the Arctic-Antarctic regions in the North and South poles of earth. Figure N0.18 shows the directions of drifting of plates.

\section{Plates moving paths}

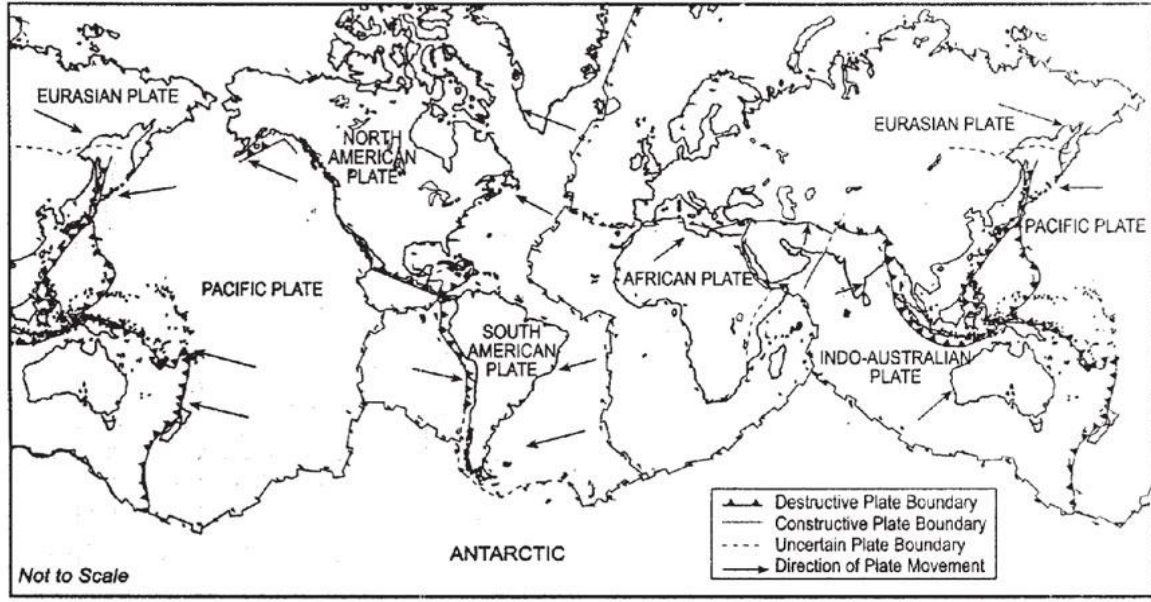

Fig. -18

Earth was a sphere, prior to the formation of ice. It could maintain the spherical shape even after it was covered by ice. The cross-sectional view of earth during ice-age is shown in fig. 19.

\section{Earth in Ice Age}

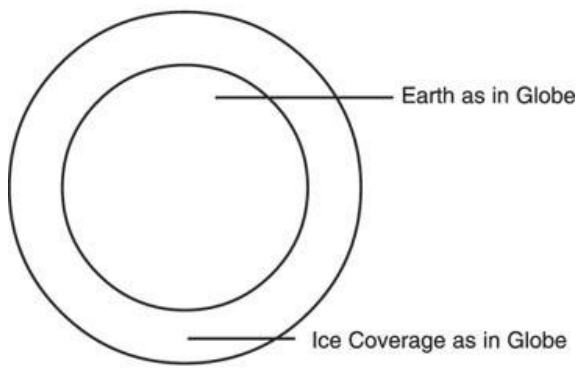

Fig. - 19

As the luminosity of sun reached 1 from 0.7 , ice started melting from the equatorial region, towards the poles. At present the luminosity is 1.4. It is continuously increasing. That is why, layers of ice melt away in Arctic and Antarctic. Figure No.20 given below, shows how the glaciers of Arctic and Antarctic keep earth in an egg-like shape. The same figure shows how the constant exertion of the gravitational forces tries to bring back earth to its spherical shape, by moving the plates. 


\section{Gravitational Force and Plates Movements}

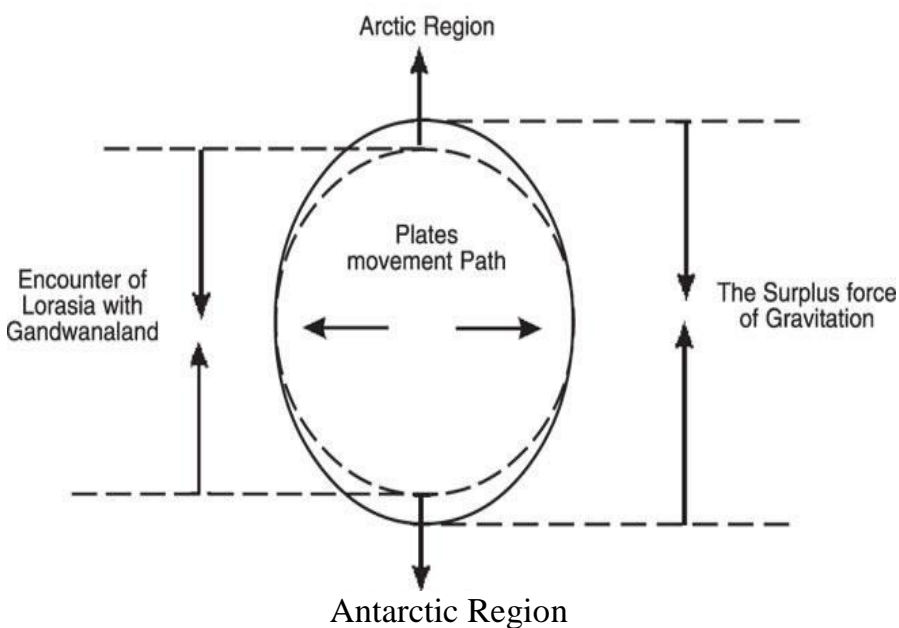

Fig.- 20

At present, the glaciers at Arctic and Antarctic regions deform the earth, by changing its spherical shape. The drifting of plates will cease, only when all the ice present on Earth melts away. During summer solstice, more ice is formed at Arctic. So, there is a possibility of annual, directional change in the drifting of plates.

If we press two opposite sides of an inflated balloon with our palms, the mid-region bulges out. Like that, the extra pressure exerted at the equatorial region by the Arctic-Antarctic poles makes the equatorial region bulge out. This results in the drifting of plates. The Himalaya was formed due to the collision between Gondwana Land and Laurasia, during such drifting. Once the luminosity of sun decreases and reaches the 0.7 level, the Ice Age will return. That time plates and continents will go back to their original positions.

\section{Summer Solstice And Winter Solstice}

Science is still silent on solstice. During summer solstice, ice melts at Arctic and glaciers are formed at Antarctic. The reverse process happens during winter solstice. But, since solar heat increases, the formation of ice decreases and melting increases. This take place, year after year. That is why, sea-level is rising continuously. During solstices, the movement of plates depends on the melting and formation of ice.

Everybody agrees that, there is a reason behind solstice. But so far, no evidence could be put forward by science - not even a distant chance in this regard.

According to the first law of Newton, unless an external disturbance affect, a moving body continues its state of motion. But this law does not have any significance within sun. Because sun moves back without any hindrance, after a certain limit.

As regarding the movement of sun, if there is no external obstruction, there must be an internal obstruction. The reason behind is that, there is a possibility of elastic gravitation in sun. Elastic gravitation means the dynamic gravitational force of matter. Even though sun appears spherical, it is actually in liquid state. Half the radius of sun is distinct from the gravitational stress region.

There must be a reason for the formation of planets from sun. It does not seem to be from an explosion of the sun. The logically correct explanation is that, some star or planet from external space may have hit sun and scattered some parts which became planets. As a result of this impact, sun moved in the opposite direction. In addition to that, there is a chance of keeping the giant star or planet which collapsed on the sun, from escaping the gravitational field of the sun. Due to the impact of the collision, the core centre of the sun moved with more speed than that of the outer region. But, the outer region pulled back the core centre by elastic gravitation. The core centre which moved backwards due to this action was again pulled back by the centre region after a certain limit. That is, along with the forward and backward motion of the core centre, due to elastic gravitational pull, the outer region follows this motion. Thus, solstice continues over time. The elasticity of liquid matter is the cause for the elastic gravitation In zero Gravity, if an object is held at the centre of a ring with elastic bands and an impact is given on it, it moves to and fro continuously. But, the ring also moves after the object without stopping. The elastic band which binds the ring and the object together, pulls back whichever moves forward. The repetition of this may be the reason behind the continuing motion. This is like the constant side-wise movement of the pendulum of a clock. This is only a hypothetical model for the cause of sun's solstice. For Further details, visit my web www.sooryapublications.com . 


\section{Reference}

[1]. WWW. Plate tectonics Thought co- Amanda briney

[2]. WWW. Plate tectonics- wikipedia

\section{About the Writer}

Dharmaj Mitra

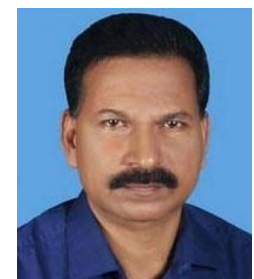

INDIA

Ph: +919446226611

dharmajmithra@gmail.com

www.sooryapublications.com 